\title{
Visual Hallucinations with Disembodiment and Self-Motion Illusion on Eye Closure after Brain Cyst Drainage: A Case Report
}

Anna Sontheimer ${ }^{1,2,+}$, Guillaume Coll ${ }^{1,2}$, Sophie Mathais ${ }^{1,3}$, François Vassal ${ }^{1,4}$, Jean-Jacques Lemaire ${ }^{1,2}$

\begin{abstract}
Background

We report the first documented case of visual hallucinations with disembodiment and selfmotion illusion on eye closure.

\section{Clinical presentation}

A 71-year-old woman was operated on for a cyst of the left centrum semiovale, adjacent to the precuneus, the posterior cingulate cortex and the splenium of corpus callosum. Two days after, she complained of visual hallucinations occurring on eye closure. She reported reminiscences of landscapes, trees and animals. She had a sensation of floating and moving, flying over the landscapes or walking on lanes. She could stop the hallucinations by opening her eyes. The hallucinations lasted for six days. Pharmacological and neurological possible origins of the hallucinations were explored.
\end{abstract}

\section{Conclusion}

No direct relation could be found between the pharmacological treatment and the hallucinations. Cerebral volumetric analysis showed a structural reorganization following cyst drainage, which might have conducted to transient hyper excitability of the surrounding structures. The release phenomenon induced by eye closure might have triggered the activation of a distributed network within associative visual, parietal and temporal cortices. The involvement of the splenium and the cingulum is discussed. Furthermore, MRI- and DTI-follow-up suggests that structural reorganization after surgery can be spread over long periods until disappearance of cyst-related deformations. Stabilization of cyst volume, near to zero, was observed after about 2 years.

\section{Keywords}

Cingulum, Disembodiment, Eye Closure, Visual Hallucinations, Self-Motion Illusion, Splenium of Corpus Callosum

\footnotetext{
'Université Clermont Auvergne, CNRS, SIGMA Clermont, Institut Pascal, Clermont-Ferrand, France

${ }^{2} \mathrm{CHU}$ Clermont-Ferrand, Service de Neurochirurgie, France

${ }^{3} \mathrm{CHU}$ Clermont-Ferrand, Service de Neurologie, France

${ }^{4} \mathrm{CHU}$ Saint-Etienne, Service de Neurochirurgie France

${ }^{\dagger}$ Author for correspondence: Anna Sontheimer, Service de Neurochirurgie, CHU Clermont-Ferrand, 58 rue Montalembert, F-63003 Clermont-Ferrand, France, Tel: 0033473752 374; Fax: 0033473751 201; email: asontheimer@chu-clermontferrand.fr
} 


\section{Case Report}

We report the first documented case of visual hallucinations associated with disembodiment and self-motion illusion, observed after brain cyst drainage. Visual hallucinations on eye closure have been previously reported [1-9], but never with disembodiment nor self-motion illusion.

\section{- Case description}

A 71-year-old right-handed woman was operated on for a periventricular benign cyst of the left centrum semiovale, facing the left precuneus, posterior cingulate cortex and the splenium of corpus callosum. The cyst was fortuitously discovered 6 years before, and remained asymptomatic until a right inferior quadranopsia appeared. The patient had a history of thyroidectomy and hypertension, and experienced migraines in the past 30 years. Preoperative biological data were within normal ranges. Endoscopic drainage was performed under propofol-induced general anaesthesia, with a navigation-guided entry point in the left superior parietal lobule. Two days after, she complained of hallucinations occurring on eye closure, within the bilateral visual field. She first reported green and red phosphenes. A few hours later, she reported vivid reminiscences of familiar landscapes, trees and animals. She didn't feel like a passive viewer, she was rather active with a sensation of moving through the environment. She felt disembodied, flying over the landscapes with a viewpoint from above, or walking on country lanes. She could stop the hallucinations by opening her eyes. She first was frightened, then get reassured. The hallucinations lasted for six days. The last days, she felt more passive and had to wait a bit after eye closure to see them. At the very end, they were limited to red filaments in her right visual field. She was all the while lucid, well oriented and not delirious. She knew the hallucinations were not real.

We hypothesized that either medications or structural modifications following surgery could have provoked the hallucinations, as she had no history of psychiatric disorders.

In addition, a 44-months MRI longitudinal follow-up was conducted.

The patient has given written informed consent for publication.

\section{Material and Methods}

Medicinal treatment and anaesthesia medication were screened to detect drugs with potential hallucinogenic effect. Periods of treatment and duration of the pharmacological effect (estimated at 5 half-lives) were compared to the hallucinatory period.

Structural modifications following surgery were investigated with cerebral volumetric analysis. MRI examination was performed the day before surgery (D-1) and seven days after (D7), corresponding to the last day of hallucinations. MRI scans were co-registered and processed using iPlan (BrainLab). D-1 and D7 MRI volumes of brain cyst and surrounding structures were measured for comparison. The precuneus was segmented on sagittal slices with the following limits: anterior, marginal segment of the cingulate sulcus; posterior, parieto-occipital fissure; superior, superior margin of the brain; inferior, subparietal sulcus; medial, midline; lateral: disappearance of subparietal sulcus. The posterior cingulate gyrus (including retrosplenial cortex) was segmented with the following limits: anterior, coronal slice through the posterior commissure; posterior: subparietal sulcus or parallel line to the curvature of the splenium; superior, cingulate and subparietal sulci; inferior: corpus callosum; medial: midline; lateral: disappearance of cingulate sulcus. The splenium of corpus callosum was segmented according to Hofer and Frahm's classification [10].

MRI follow-up was conducted at eight (M8), fifteen (M15), thirty-nine (M39) and fortyfour (M44) months after surgery. The MRI examination included DTI in 20 directions at D-1, M8 and M44. Long-term follow-up analysis was conducted with brain cyst volume measurement at each time point and with DTI-based tractography applied to the corpus callosum and the cingulum when data were available.

\section{Results}

Four potential hallucinatory drugs were identified: hydroxyzine $(50 \mathrm{mg} ; 2$ preoperative doses at $\mathrm{D}-1$ ), oxycodone ( $5 \mathrm{mg} ; 2$ postoperative doses at D0 - day of surgery, and D1), tramadol (100 mg at D1 and D2) and metoprolol-tartrate (200 mg per day, background therapy). None of them was concomitant with the hallucinations (Figure 1).

Volumetric analysis between the day before and seven days after surgery showed a decrease of the cyst $(-38 \%)$ and an increase of neighbouring structures, as a result of decompression: splenium 
Visual Hallucinations with Disembodiment and Self-Motion Illusion on Eye Closure after Brain Cyst Case Report Drainage: A Case Report

of corpus callosum $(+21 \%)$, left posterior cingulate cortex $(+20 \%)$ and left precuneus (+13\%) (Figure 2). Right-hemispheric variations were negligible (Table $\mathbf{1})$.

Long term MRI follow-up analysis showed a decrease of cyst volume over time, from 15.57 $\mathrm{cm}^{3}$ (D-1) to $9.64(\mathrm{D} 7)$ to $2.4(\mathrm{M} 8)$ to 1.21
(M15) to $0.15 \mathrm{~cm}^{3}$ (M39 and M44). DTI-based tractography of corpus callosum and cingulum at D-1, M8 and M44 showed that white matter tracts of the splenium and left cingulum were still deviating eight months after surgery, whereas they went back to a regular shape at the end of the follow-up (Figure 3).

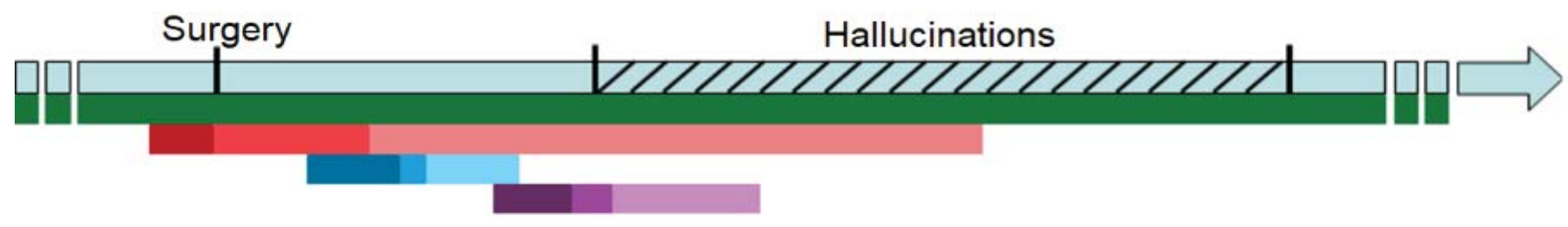
Metropolol-tartrate
Oxycodone
Hydroxyzine
Tramadol

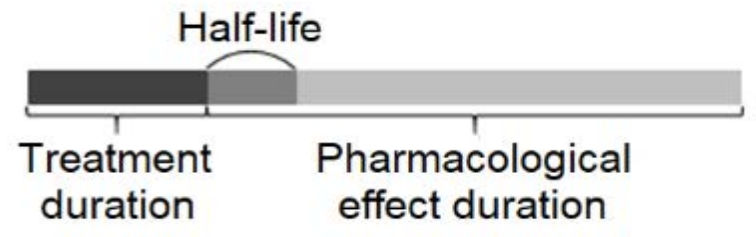

Figure 1: Timeline of the surgery, the hallucinatory period and the pharmacological treatment.
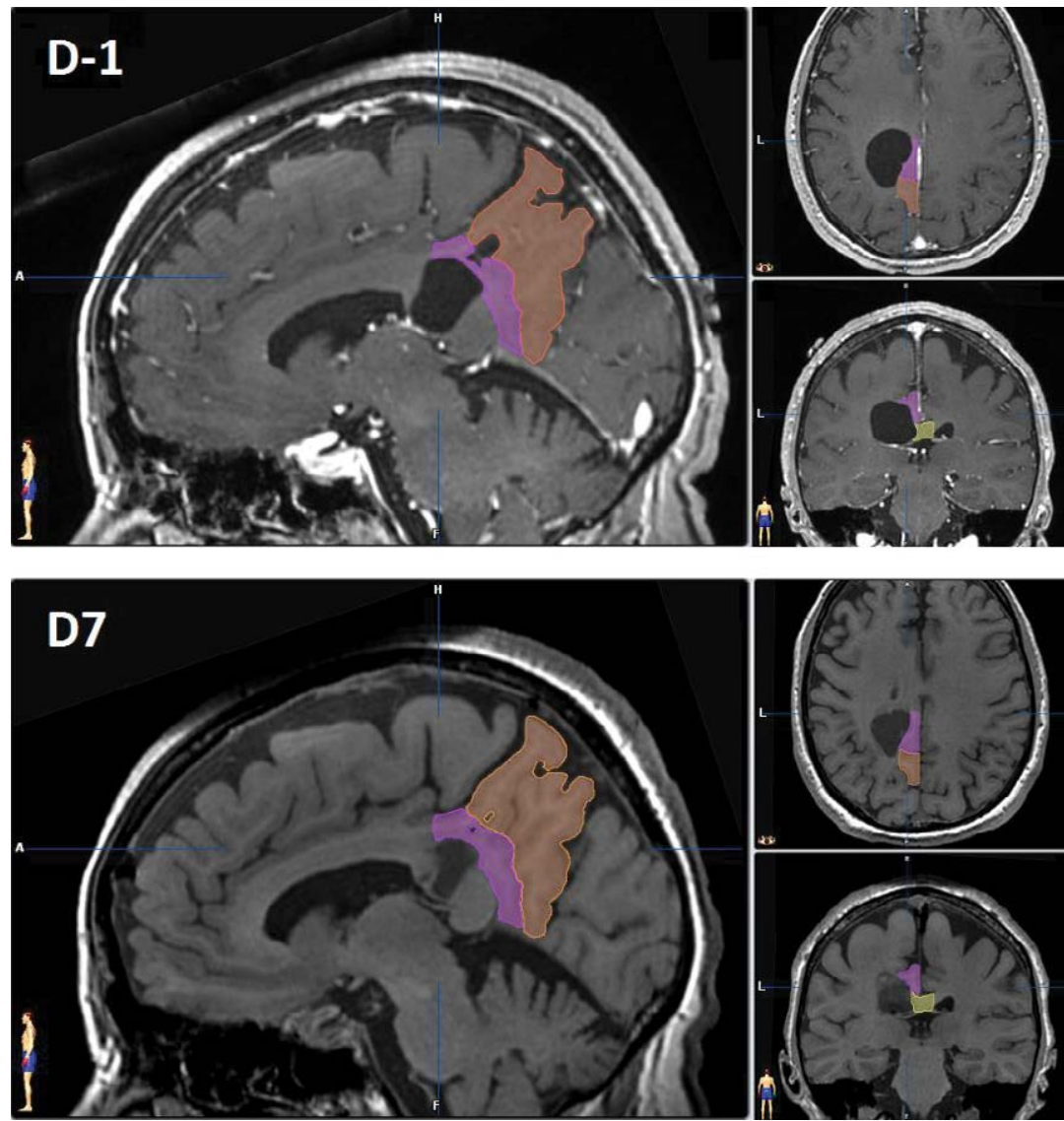

Figure 2: Contouring of the left precuneus (orange), the left posterior cingulate cortex (pink) and the splenium of corpus callosum (yellow), for volumetric analysis. (D-1) contrast-enhanced T1-weighted MRI acquired the day before surgery. (D7) T1-weighted MRI acquired the last day of the hallucinations. 


\section{Case Report Anna Sontheimer}

Table 1: Volumetric analysis: Volumes of brain cyst and surrounding structures the day before (D-1) and seven days after (D7)
surgery, expressed in $\mathbf{c m}^{3}$.
\begin{tabular}{|l|l|l|l|l|l|}
\hline cyst & $\begin{array}{l}\text { splenium of the corpus } \\
\text { callosum }\end{array}$ & $\begin{array}{l}\text { left posterior cingulate } \\
\text { gyrus }\end{array}$ & left precuneus & $\begin{array}{l}\text { right posterior cingulate } \\
\text { gyrus }\end{array}$ & right precuneus \\
\hline 15.57 & 2.665 & 3.296 & 9.106 & 3.342 & 9.189 \\
\hline 9.643 & 3.231 & 3.947 & 10.318 & 3.429 & 9.503 \\
\hline
\end{tabular}
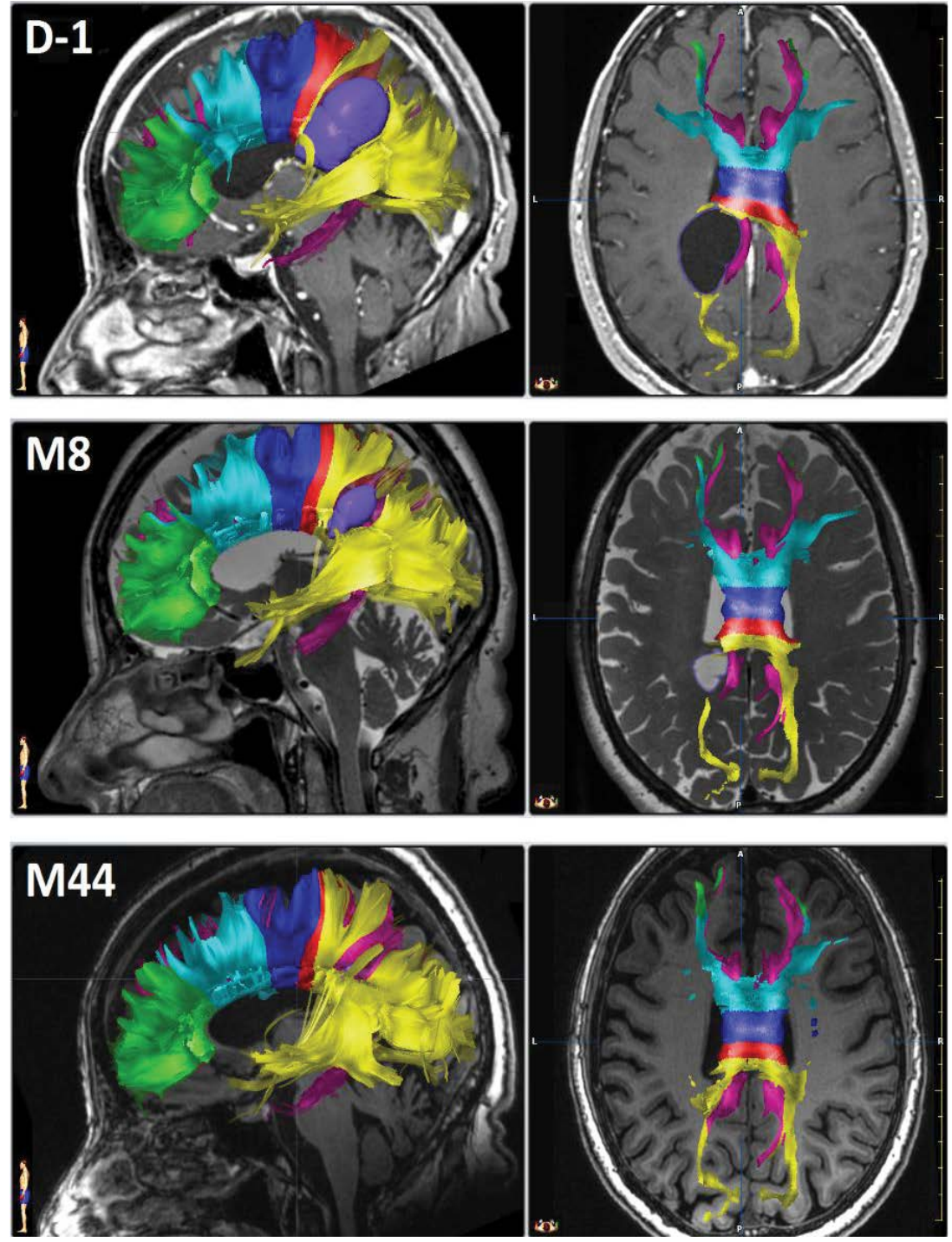

Figure 3: DTI-based tractography of bilateral cingulum (pink) and of the corpus callosum segmented according to connected areas ${ }^{8}$ : prefrontal (green), premotor and supplementary motor (cyan), motor (blue), sensory (red), occipital, parietal and temporal (yellow). Cyst contours are shown in violet. (D-1) contrast-enhanced T1-weighted MRI acquired the day before surgery. (M8) T2-weighted MRI acquired 8 months after. (M44) T1-weighted MRI acquired 44 months after.

\section{Outcome}

Five years after cyst drainage, the patient did not recover from quadranopsia. She had no recurrence of the hallucinations.

\section{Discussion}

Previous cases of visual hallucinations occurring on eye closure have been reported following hip surgery under general anaesthesia [1], local anaesthesia with lidocaine [2,3], atropine administration [4], cardiovascular surgery [5,6], traumatic subdural hematoma in the left occipital region [7], trans-sphenoidal adenomectomy [8] and posterior callosal stroke [9]. The triggering of hallucinations by eye closure can be linked to the "cortical release phenomenon" due to visual 
deafferentation in Charles-Bonnet Syndrome [7]. The eye closure leads to the disruption of afferent visual input, and thus allows the release of visual hallucinations due to spontaneous endogenous neuronal activity in occipital visual cortices. Nonetheless, none of the previously described cases was associated with neither disembodiment nor self-motion illusion. Disembodiment is a feature of out-of-body experiences, involving the temporoparietal junction [11]. Vection or illusion of self-motion can be induced by the stimulation of the precuneus $[12,13]$. The patient reported visual reminiscences, which can be induced by the stimulation of rhinal cortices within the medial temporal lobe [14]. Thus, the symptomatology of the hallucinations indicates a common involvement of occipital, parietal and temporal cortices. The hallucinations appeared mostly bilaterally, implying a recruitment of both hemispheres. The splenium of corpus callosum connects occipital, parietal and temporal cortical areas [10] (see yellow tracts in Figure 3) and thereby is a good candidate in the genesis of these atypical hallucinations. The cingulum, connecting cingulate gyrus, precuneus and medial temporal lobe, might also be involved. Volumetric analysis before and after cyst drainage showed an increase of the cyst surrounding structures. This structural reorganization might have conducted to a transient hyper excitability. The release phenomenon induced by eye closure might have triggered the activation of a distributed network within associative visual, parietal and temporal cortices.

Visual hallucinations are relatively frequent in patients with acute stroke [15], and visual hallucinations on eye closure have been already described in a case of posterior callosal stroke [9]. However, in the present case, it seems unlikely that the hallucinations were related to stroke or epilepsy induced stroke, as the MRI scans acquired the day before surgery and the last day of the hallucinations showed no evidence of stroke. Electroencephalogram was not performed during the hallucinatory period, but the fact that the hallucinations occurred only during eye closure and that there was no postictal-like confusion once she opened her eyes tends to reject the hypothesis of epileptic seizure [16].

No direct relation could be found between the pharmacological treatment, including anaesthesia medication, and the hallucinations. Yet, Tramadol might have exacerbated the phenomenon, as previously observed [17].

Long-term follow-up showed a stabilization of cyst volume, near to zero, between 15 and 39 months after endoscopic surgery. DTIbased tractography analysis showed that the white matter tracts of splenium and cingulum were still deviating eight months after surgery, whereas they went back to a regular shape at the end of the follow-up. These findings suggest that structural reorganization after endoscopic cyst removal can be spread over long periods until disappearance of cyst-related deformations.

A particular attention should be paid to occurrence of hallucinations after neurosurgical procedures in the regions of the precuneus, the posterior cingulate cortex and the splenium of corpus callosum. Patients should be warned, because hallucinations can be very stressinducing.

\section{Conflict of Interest}

The authors declares no conflict of interest

\section{Disclosure of Funding}

Fondation de l'Avenir ("Deroche Legacy” Grant BO4-001; wages of Anna Sontheimer). 


\section{Case Report Anna Sontheimer}

\section{References}

1. Otomo S, Sugita M, Yano T. Visual hallucinations on eye closure after orthopedic surgery under general anesthesia. J. Anesth 22(4), 439-442 (2008).

2. Fisher $\mathrm{CM}$. Visual hallucinations and racing thoughts on eye closure after minor surgery. Arch. Neurol 48(10), 1091-1092 (1991).

3. Weinschenk K, Schwartz AC. A case report of closed-eye visual hallucinations. Psychosomatics 52(1), 86-87 (2011).

4. Fisher CM. Visual hallucinations on eye closure associated with atropine toxicity. A neurological analysis and comparison with other visual hallucinations. Can. J. Neurol. Sci 18(1), 18-27 (1991).

5. Eissa A, Baker RA, Knight JL. Closed-eye visual hallucinations after coronary artery bypass grafting. J. Cardiothorac. Vasc. Anesth 19(2), 217-219 (2005).

6. Laloux $P$, Osseman M. Visual hallucinations on eye closure after cardiovascular surgery. J. Clin. Neuroophthalmol 12(4), 242-244 (1992).
7. Huang CW, Chen $\mathrm{HH}$, Chen NC, et al. Complex visual hallucinations triggered by eye closure and condition-specific release phenomenon. Neuropsychiatry 6(6), 298302 (2016).

8. Park JH, Ahn JH, Park JB, et al. Charles Bonnet Syndrome Following TransSphenoidal Adenomectomy without Optic Nerve Atrophy. Psychiatry. Investig 13(5), 577-579 (2016).

9. Ghosh GN, Wycoco V, Ghosh S. Transient Visual Hallucinations due to Posterior Callosal Stroke. J. Stroke. Cerebrovasc. Dis 24(6), 147-148 (2015)

10. Hofer S, Frahm J. Topography of the human corpus callosum revisited-comprehensive fiber tractography using diffusion tensor magnetic resonance imaging. Neurolmage 32(3), 989-994 (2006).

11. Blanke O, Mohr C. Out-of-body experience, heautoscopy, and autoscopic hallucination of neurological origin Implications for neurocognitive mechanisms of corporeal awareness and self-consciousness. Brain. Res. Rev 50(1), 184-199 (2005).

12. Kovacs G, Raabe M, Greenlee MW. Neural correlates of visually induced self-motion illusion in depth. Cereb. Cortex 18(8), 1779 1787 (2008).

13. Wiest G, Zimprich F, Prayer D, et al. Vestibular processing in human paramedian precuneus as shown by electrical cortical stimulation. Neurology 62(3), 473-475 (2004).

14. Bartolomei F, Barbeau E, Gavaret M, et al Cortical stimulation study of the role of rhinal cortex in deja vu and reminiscence of memories. Neurology 63(5), 858-864 (2004).

15. Morenas-Rodriguez E, Camps-Renom $P$, Perez-Cordon A, et al. Visual Hallucinations in Patients with Acute Stroke: A Prospective Exploratory Study. Eur. J. Neurol 24(5), 734740 (2017).

16. Chung SS, Gerber P, Kirlin KA. Ictal eye closure is a reliable indicator for psychogenic nonepileptic seizures. Neurology 66(11), 1730-1731 (2006).

17. Mascaro J, Formiga F, Pujol R. CharlesBonnet syndrome exacerbated by tramadol. Aging. Clin. Exp. Res 15(6), 518-519 (2003) 